\title{
Numerical Simulation of Hypersonic Flow around a Triangular Wing
}

\author{
P. Silvestrov ${ }^{1}$, I.A. Kryukov ${ }^{1,2}$, B.V. Obnosov ${ }^{1}$ \\ ${ }^{1}$ Tactical Missiles Corporation, Korolev, Moscow district, 141080, Russia \\ ${ }^{2}$ Institute for Problems in Mechanics of the Russian Academy of Sciences, \\ Moscow, 119526, Russia \\ pavelsilvestrov@yandex.ru
}

\begin{abstract}
Results of numerical study of hypersonic flow around model of triangular wing are presented. Effects of varying angle of attack on the hypersonic flow field and on the surface properties were investigated. The integral aerodynamic properties, lift coefficients, drag coefficients for different angles of attack are done. Results are compared with experimental data.
\end{abstract}

Keywords: triangular wing, gas dynamics, mathematic modelling, computational aerodynamics, program complex, unstructured grids.

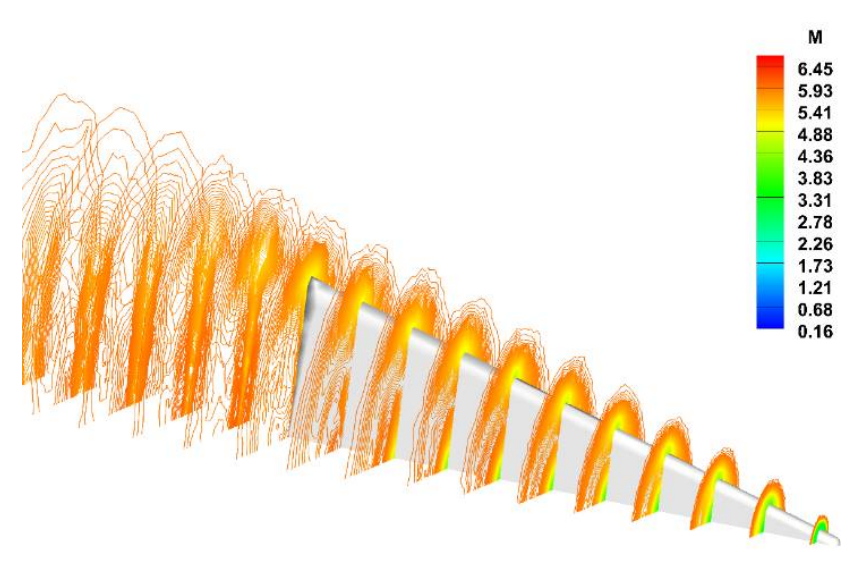

Three-dimensional flow field at $\mathrm{M}=6$

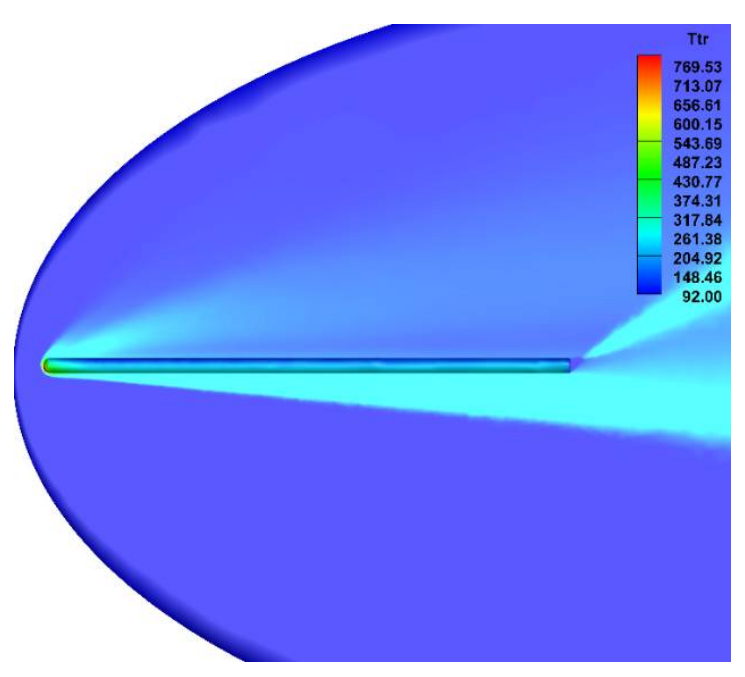

The distribution of temperature on the surface of a wing and in cross-sections at $\alpha=25^{\circ}$ 
УДК 533.6.01+519.6

\title{
Численное моделирование гиперзвукового обтекания треугольного крыла
}

\author{
П.В. Сильвестров ${ }^{1}$, И.А. Крюков ${ }^{1,2}$, Б.В. Обносов ${ }^{1}$ \\ ${ }^{1}$ Корпорациия «Тактическое ракетное вооружение», \\ Московская обл., Королев, 141080, ул. Ильича, 7 \\ ${ }^{2}$ Институт проблем механики им. А.Ю. Иилинского Российской академии наук, \\ Москва, 119526, проспект Вернадского, 101-1 \\ pavelsilvestrov@yandex.ru
}

\begin{abstract}
Аннотация
Рассмотрена задача численного моделирования внешнего гиперзвукового обтекания треугольного крыла с затупленными передними кромками. Методами вычислительной аэродинамики исследовалось возмущенное поле течения и теплофизические процессы во всей области от головной ударной волны до дальнего следа. Изучалась конфигурация ударных волн, образующихся при обтекании модели, а также фиксировались области взаимодействия ударных волн с поверхностью. Исследовалось влияние угла атаки и числа Маха на поле течения, аэродинамические и тепловые характеристики поверхности крыла. Также для каждого режима полета определены интегральные аэродинамические характеристики, коэффициенты подъемной силы $C_{y}$, коэффициенты силы лобового сопротивления $C_{x}$. Проведено сравнение результатов численного моделирования с результатами численного моделирования ЦАГИ.
\end{abstract}

Ключевые слова: треугольное крыло, газовая динамика, математическое моделирование, вычислительная аэродинамика, программные комплексы, неструктурированные сетки.

\section{1. Введение}

Изучение гиперзвуковых пространственных течений вязкого теплопроводного газа является актуальной проблемой современной аэродинамики и представляет значительный интерес с точки зрения приложений к разработке нового поколения высокоскоростных летательных аппаратов. Интенсивные исследования в этой области проводились в основном с использованием асимптотических методов и теории пограничного слоя [1]. В рамках такого подхода были выявлены основные параметры, управляющие рассматриваемым классом течений, изучены эффекты вязко-невязкого взаимодействия, распространения возмущений давления и возникновения отрыва в ламинарном пограничном слое, построены автомодельные решения, рассмотрены задачи теплообмена. В то же время целый ряд наблюдаемых в опытах явлений еще не получил адекватного объяснения.

Для решения задачи разработки перспективных гиперзвуковых летательных аппаратов (ГЛА), необходимо проведение как экспериментальных, так и расчетно-теоретических исследований аэродинамических характеристик, и особенностей обтекания интегральных компоновок гиперзвуковых аппаратов с прямоточными воздушно-реактивными двигателями. Проведение подобных летных и наземных испытаний требует очень больших затрат, поэтому в настоящее время возрастает роль численного моделирования в разработке гиперзвуковых летательных аппаратов. При проектировании перспективных летательных аппаратов, как правило, проводятся численные исследования обтекания предполагаемых конфигураций планера, которые существенно сокращают затраты на проведение испытаний в аэродинамических трубах. При этом в процессе расчета возможно определение всех параметров течения, 
тогда как в эксперименте удается измерять лишь отдельные газодинамические величины. Это становится особенно актуальным при моделировании гиперзвуковых условий полета.

Вопросам исследования гиперзвуковой аэродинамики посвящено большое количество работ, например [2-3]. Проблеме моделирования гиперзвукового обтекания сложных тел посвящены работы, например [4-5] на структурированных сетках и [6-7] на неструктурированных сетках. Решение задач аэродинамики на гиперзвуковых скоростях для тел упрощенной формы рассматриваются в работах [8-9], для тел в форме крыла - в [10-13].

В [14] проведено подробное исследование структуры течения около треугольного крыла на основе численного решения полных уравнений Навье - Стокса для ламинарного режима с использованием расчетных сеток на 2, 4, 10 и 50 млн узлов и приведено сравнение с результатами экспериментальных исследований в ударной трубе ЦАГИ УТ-1М.

В данной статье рассматривается вопрос возможности проведения подобных исследований с менее подробными сетками на разных углах атаки и числах Маха.

\section{2. Постановка задачи}

\section{1. Геометрическая модель}

Для численного моделирования процесса обтекания была создана компьютерная модель поверхности треугольного крыла с углом стреловидности передних кромок $\chi=75^{\circ}$, толщиной 16 мм, радиусом затупления кромок и носка 8 мм, длиной $l=570$ мм (рис. 1). В качестве прообраза выбраны эскизы, опубликованные в [14] (рис. 2).

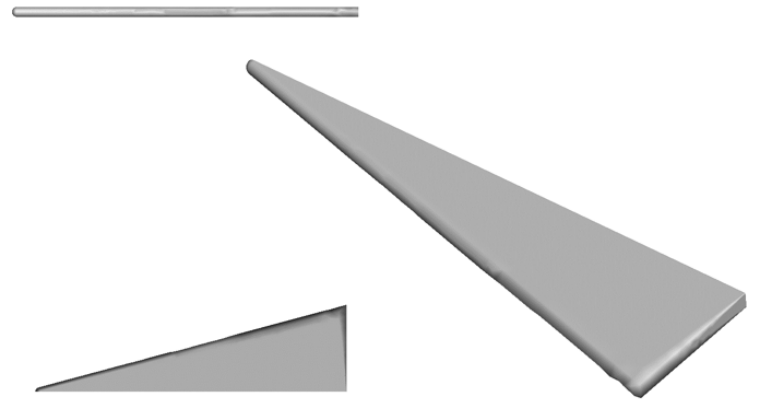

Рис. 1 Построенная геометрия модели

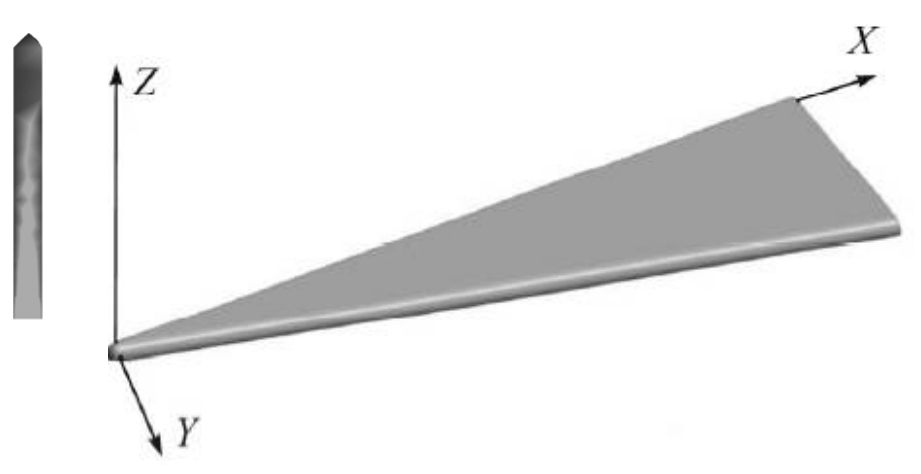

Рис. 2 Исходная геометрия модели [14]

\section{2. Построение расчетной сетки}

В настоящей работе моделирование трехмерного поля течения около треугольного крыла проводилось с использованием неструктурированных тетраэдральных сеток. На рис. 3-4 представлены различные фрагменты трехмерной неструктурированной расчетной сетки, применявшейся для моделирования внешнего обтекания объекта.

Из рис. 3, а хорошо видна степень сгущения ее узлов к обтекаемой поверхности вдоль плоскости симметрии. Размерность сетки составляла примерно 1600000 тетраэдральных элементов. Радиус затупления кромок и носка составлял 8 мм. 


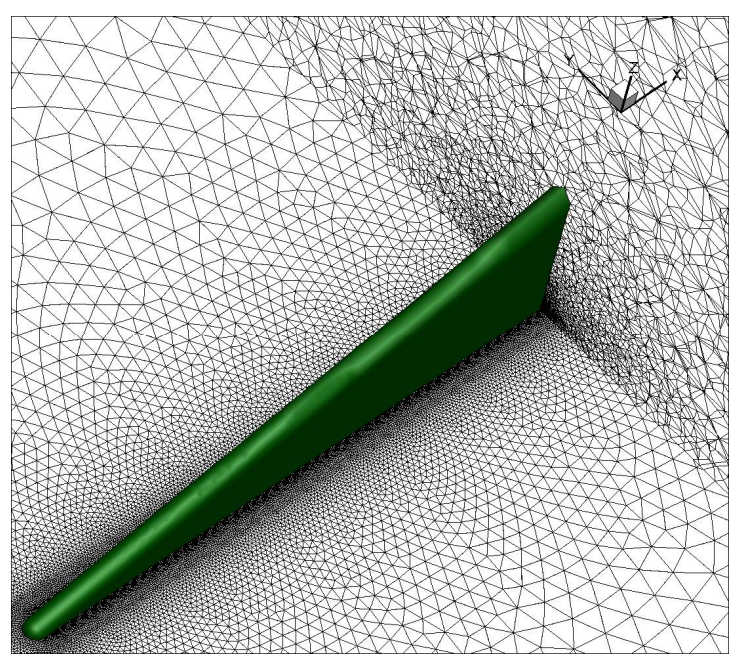

a)

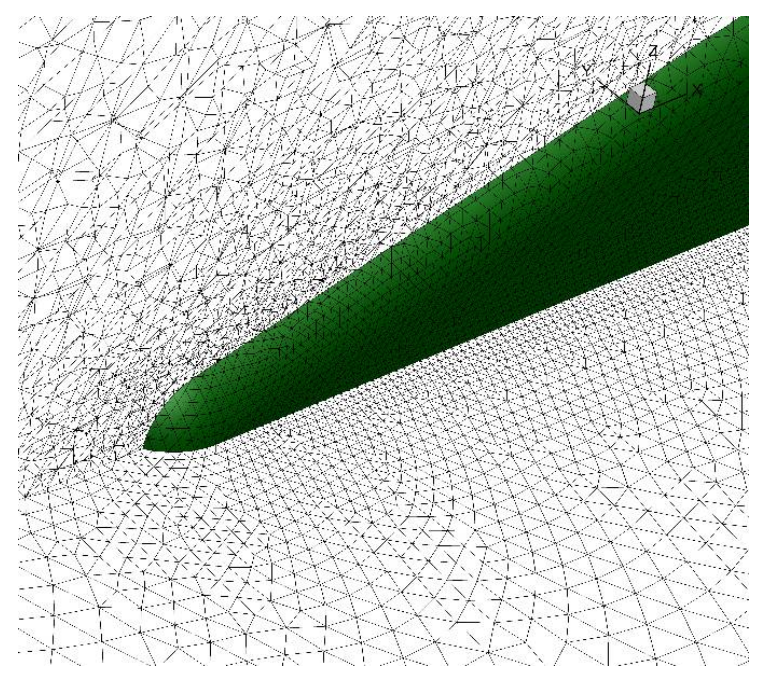

б)

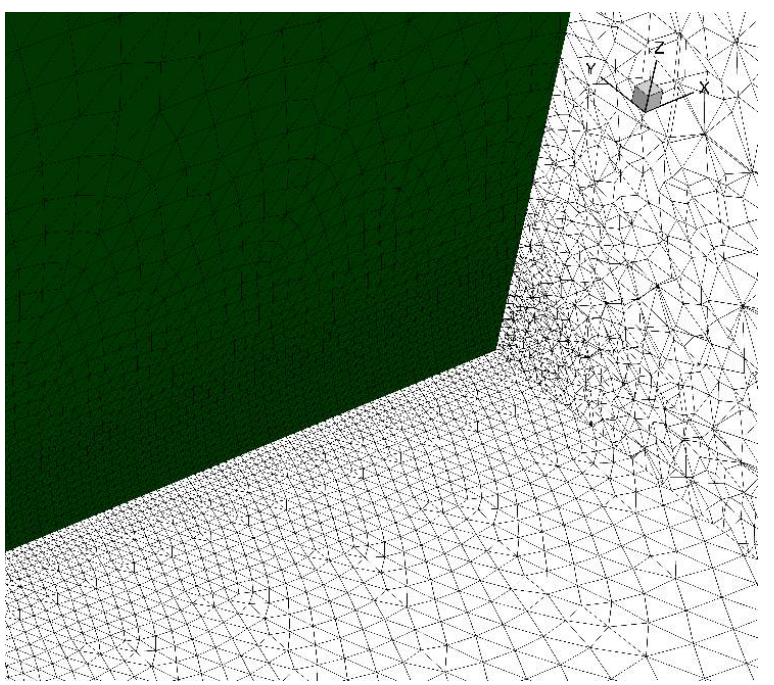

в)

Рис. 3. Объемная расчетная сетка

\section{3. Параметры набегающего потока}

Параметры набегающего потока воздуха приведены в табл. 1. Расчеты выполнены для чисел Маха $\mathrm{M}=\{4,6\}$ и углов атаки $0^{\circ}, 10^{\circ}$ и $25^{\circ}$. Для проведения сравнения исходные данные для набегающего потока при $\mathrm{M}=6$ взяты из [14]. В [14] не приведены данные для $\mathrm{M=4}$, поэтому и в этом случае в качестве параметров набегающего потока были взяты данные для $\mathrm{M}=6$.

\section{Таблица 1}

Исходные данные для расчета и свойства набегающего потока

\begin{tabular}{|c|c|}
\hline Наименование параметра & Значение \\
\hline Число Маха & 4,6 \\
\hline Угол атаки, градусы & $0,10,25$ \\
\hline Температура стенки, K & 290 \\
\hline Температура набегающего потока, K & 91.46 \\
\hline Давление набегающего потока, Па & 1283.5 \\
\hline Плотность набегающего потока, кг/м³ & 0.0489 \\
\hline Скорость звука, м/с & 192 \\
\hline Динамическая вязкость, кг/(мс) & $0.766 \times 10^{-5}$ \\
\hline
\end{tabular}




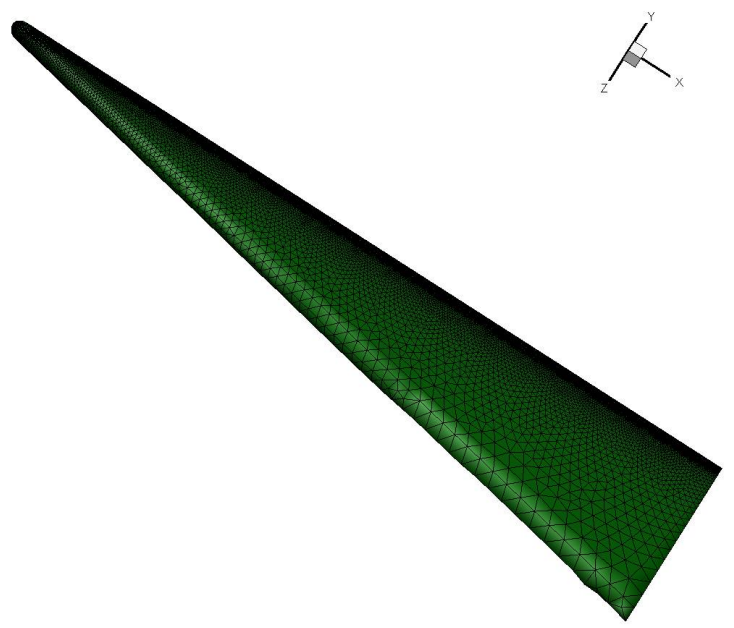

a)

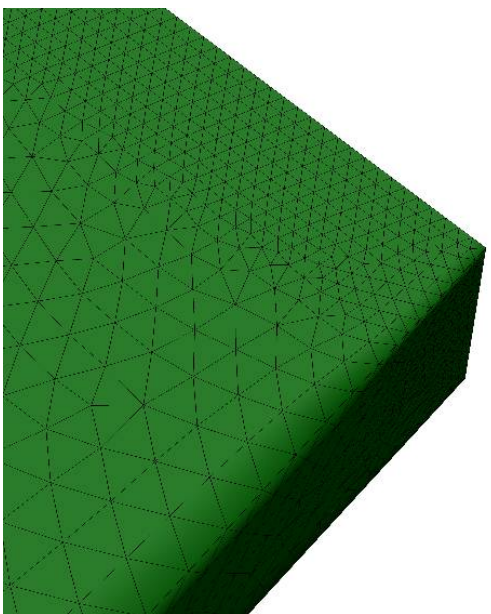

в)

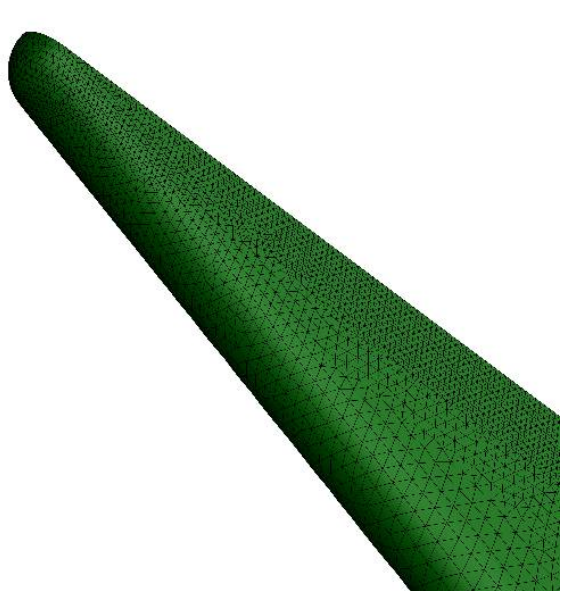

б)

Рис. 4. Поверхностная расчетная сетка

\section{3. Математическая постановка задачи}

\section{1. Исходная система уравнений}

Пространственное обтекание модели ГЛА описывается трехмерной нестационарной системой уравнений Навье-Стокса, которая записана в векторной форме [15]

$$
\frac{\partial w}{\partial t}+\frac{\partial F^{x}(w)}{\partial x}+\frac{\partial F^{y}(\omega)}{\partial y}+\frac{\partial F^{z}(\omega)}{\partial z}=\frac{\partial G^{x}(\omega)}{\partial x}+\frac{\partial G^{y}(\omega)}{\partial y}+\frac{\partial G^{Z}(\omega)}{\partial z}
$$

В данном выражении:

$\omega=(\rho, \rho u, \rho v, \rho w, \rho E)^{T}$ - вектор консервативных переменных;

$F^{x}=\left(\rho u, \rho u^{2}+p, \rho u v, \rho u w, \rho u E+p u\right)^{T}, F^{y}=\left(\rho v, \rho u v, \rho v^{2}+p, \rho v w, \rho v E+p v\right)^{T}$,

$F^{z}=\left(\rho w, \rho u w, \rho v w, \rho w^{2}+p, \rho w E+p w\right)^{T}-$ компоненты вектора конвективного потока;

$G^{x}=\left(0, \tau_{x x}, \tau_{y x}, \tau_{z x}, u \tau_{x x}+v \tau_{y x}+w \tau_{z x}-q_{x}\right)^{T}, G^{y}=\left(0, \tau_{x y}, \tau_{y y}, \tau_{z y}, u \tau_{x y}+v \tau_{y y}+w \tau_{z y}-q_{y}\right)^{T}$,

$G^{z}=\left(0, \tau_{x z}, \tau_{y z}, \tau_{z z}, u \tau_{x z}+v \tau_{y z}+w \tau_{z z}-q_{z}\right)^{T}-$ компоненты вектора вязкого потока; 
$\rho$ - плотность; $u, v, w$ - компоненты вектора скорости; $p$ - давление; $E$ - удельная полная энергия газа; $q_{x}, q_{y}, q_{z}$ - компоненты вектора теплового потока; $\tau_{\alpha \beta}$ - компоненты тензора вязких напряжений $(\alpha=x, y, z) ;(\beta=x, y, z)$ описываемые в виде

$$
\begin{gathered}
\tau_{x x}=\mu\left(\frac{4}{3} \frac{\partial u}{\partial x}-\frac{2}{3} \frac{\partial v}{\partial y}-\frac{2}{3} \frac{\partial w}{\partial z}\right) ; \tau_{y y}=\mu\left(\frac{4}{3} \frac{\partial v}{\partial y}-\frac{2}{3} \frac{\partial u}{\partial x}-\frac{2}{3} \frac{\partial w}{\partial z}\right) ; \quad \tau_{z z}=\mu\left(\frac{4}{3} \frac{\partial w}{\partial z}-\frac{2}{3} \frac{\partial u}{\partial x}-\frac{2}{3} \frac{\partial v}{\partial y}\right) \\
\tau_{x y}=\tau_{y x}=\mu\left(\frac{\partial v}{\partial x}+\frac{\partial u}{\partial y}\right) ; \quad \tau_{y z}=\tau_{z y}=\mu\left(\frac{\partial w}{\partial y}+\frac{\partial v}{\partial z}\right) ; \quad \tau_{x z}=\tau_{z x}=\mu\left(\frac{\partial u}{\partial z}+\frac{\partial w}{\partial x}\right)
\end{gathered}
$$

Здесь $\mu$ - коэффициент динамической вязкости.

В качестве замыкающих соотношений используются: $p=(\gamma-1) \rho \mathrm{U}=(\gamma-1) \rho\left[E-0.5\left(u^{2}+v^{2}+w^{2}\right)\right]-$ уравнение состояния совершенного газа; $U=c_{V} T-$ калорическое уравнение состояния;

$E=\frac{p}{(\gamma-1) \rho}+\frac{u^{2}+v^{2}+w^{2}}{2}-$ выражение для полной энергии; $q=-\lambda \nabla T-$ закон Фурье.

Здесь $U$ - внутренняя энергия газа; $c_{V}$ - удельная теплоемкость газового потока; $T$ - температура; $\lambda$ - коэффициент теплопроводности.

Так как в [14] рассматривается ламинарное течение, то в данной работе используется математическая модель для ламинарного режима течения без моделей турбулентности.

Постановка граничных условий осуществлялась следующим образом. На входе в расчетную область задавались условия невозмущенного потока

$$
E_{\text {enter }}=E_{\infty}=\frac{p_{\infty}}{(\gamma-1) \rho_{\infty}}+\frac{u_{\infty}{ }^{2}+v_{\infty}{ }^{2}+w_{\infty}{ }^{2}}{2}
$$

Здесь $\rho_{\infty}, u_{\infty}, v_{\infty}, w_{\infty}, E_{\infty}, p_{\infty}$ - параметры в набегающем потоке.

На выходной границе расчетной области реализовывались условия отсутствия градиентов параметров потока в направлении единичной нормали к поверхности границы:

$$
\left.\frac{\partial \rho}{\partial n}\right|_{\text {exit }}=0 ;\left.\quad \frac{\partial u}{\partial n}\right|_{\text {exit }}=\left.\frac{\partial v}{\partial n}\right|_{\text {exit }}=\left.\frac{\partial w}{\partial n}\right|_{\text {exit }}=0 ;\left.\quad \frac{\partial E}{\partial n}\right|_{\text {exit }}=0
$$

Здесь $n$ - единичная нормаль к поверхности границы.

На стенке летательного аппарата задавались условия прилипания. Температура стенки поддерживалась постоянной

$$
\left.\frac{\partial \rho}{\partial n}\right|_{\text {wall }}=0 ;\left.\quad u^{n}\right|_{\text {wall }}=\left.v^{n}\right|_{\text {wall }}=\left.w^{n}\right|_{\text {wall }}=0 ;\left.\quad \frac{\partial T}{\partial n}\right|_{\text {wall }}=0 ;\left.\quad T\right|_{\text {wall }}=T_{\text {wall }}
$$

Здесь $T_{\text {wall }}$ - температура стенки.

В силу симметрии летательного аппарата расчеты производились для половины расчетной области. В плоскости симметрии задавались соответствующие граничные условия

$$
\left.\frac{\partial \rho}{\partial n}\right|_{\text {symm }}=0 ;\left.\quad u^{n}\right|_{\text {symm }}=\left.v^{n}\right|_{\text {symm }}=\left.w^{n}\right|_{\text {symm }}=0 ;\left.\quad \frac{\partial T}{\partial n}\right|_{\text {symm }}=0
$$

Задача моделирования пространственного обтекания поверхности ГЛА сложной формы состоит в расщеплении исходной системы уравнений по физическим процессам. 
Решение задачи, если оно существует, получается в результате установления. В данном методе не используется выделение поверхностей разрыва. Основные уравнения газовой динамики записываются в виде законов сохранения, что обеспечивает дивергентность и консервативность получаемых при аппроксимации разностных схем. Это обеспечивает достаточно высокую точность вычислений, т.к. законы сохранения будут выполняться как для элементарной ячейки, так и для расчетной области. Метод расщепления по физическим процессам может быть подразделен на следующие этапы:

- Эйлеров этап - учитываются только процессы, связанные с действием сил давления; Рассчитываются промежуточные значения параметров газа;

- $\quad$ Лагранжев этап - рассматриваются конвективные эффекты и явления переноса, находятся потоки массы через границы расчетных ячеек;

- Заключительный этап - на основе законов сохранения массы, импульса и энергии определяются окончательные значения параметров потока для каждого элемента и системы в целом.

Метод является явным и обладает первым порядком аппроксимации по времени и вторым по пространству.

\section{4. Результаты численных расчетов}

Расчеты проводились с использованием авторского компьютерного кода UST3D (UnStructured Tetrahedron 3-Dimensional), который предназначен для расчета аэродинамики и пространственных распределений газодинамических параметров при обтекании элементов конструкции или полной компоновки гиперзвукового летательного аппарата длительного атмосферного полета. Компьютерный код был разработан Суржиковым С.Т. в ИПМех РАН [16-17].

На рис. 4 дано изображение трехмерного поля течения (распределение числа Маха) около треугольного крыла при $\mathrm{M}=6$ и $\alpha=0^{\circ}$. Рисунок дает представление о сложности структуры ударных волн, образующихся при обтекании крыла, но не является в достаточной мере информативным. Для более детального изучения характера взаимодействия ударных волн рассмотрим картину течения в центральном осевом сечении.

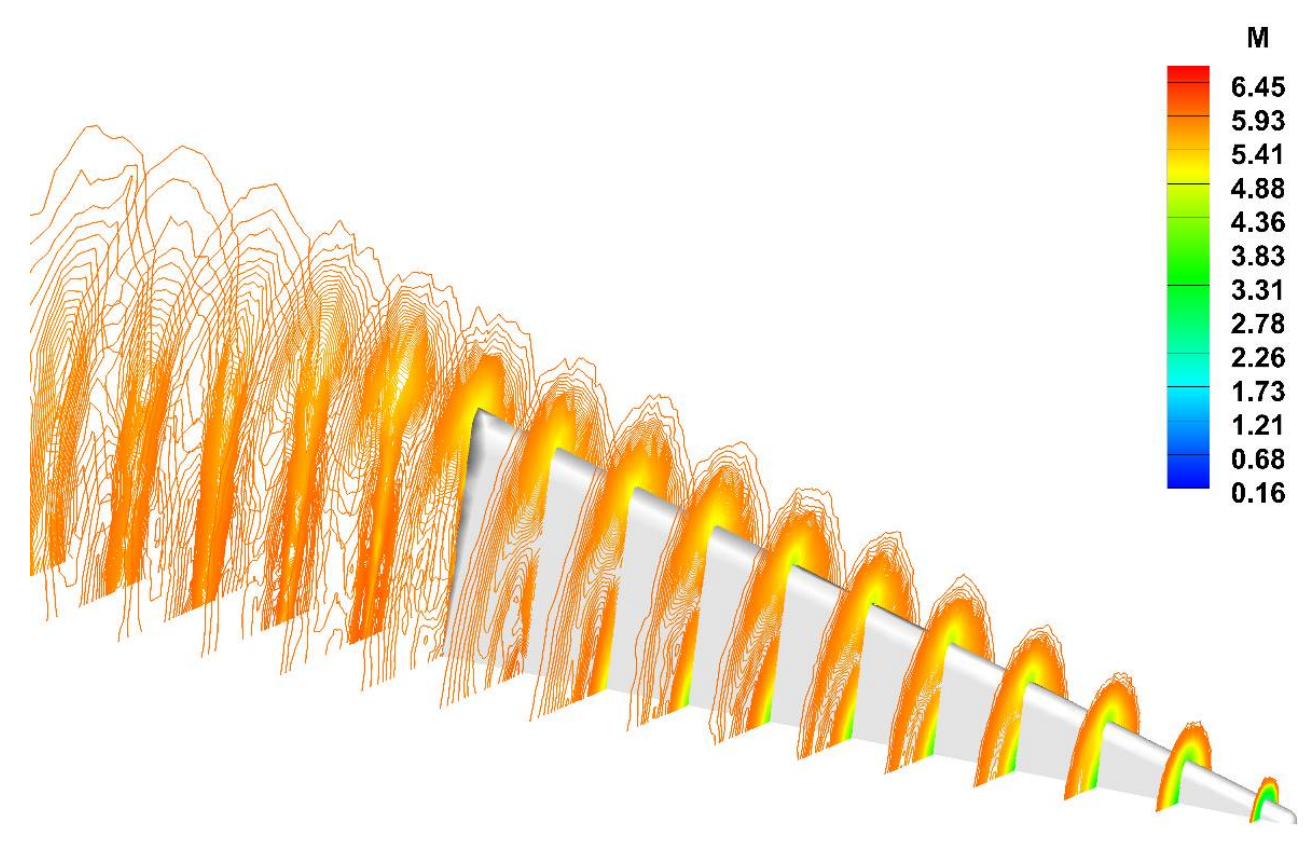

Рис. 4. Трехмерное поле течения при $\mathrm{M}=6$ 
По расчетным данным, представленным на рис. $5, a-6$ можно проследить изменение полей чисел Маха и конфигурации поля течения с изменением угла атаки в центральном осевом сечении.

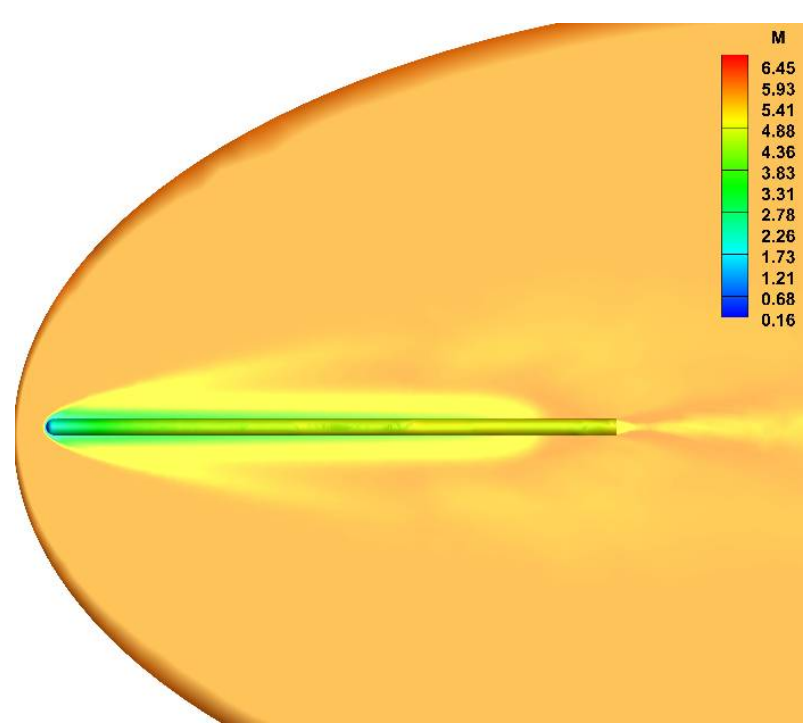

a)

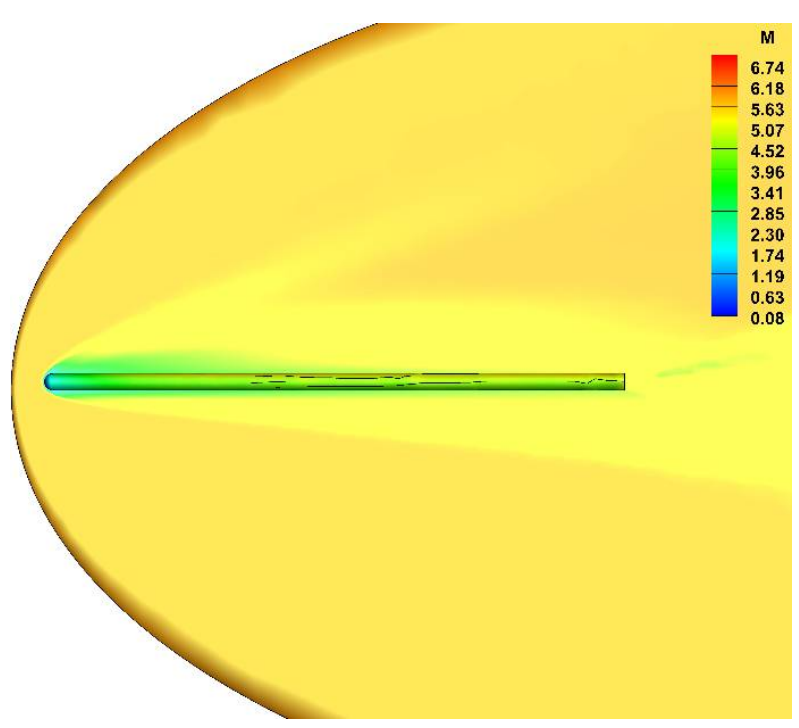

б)

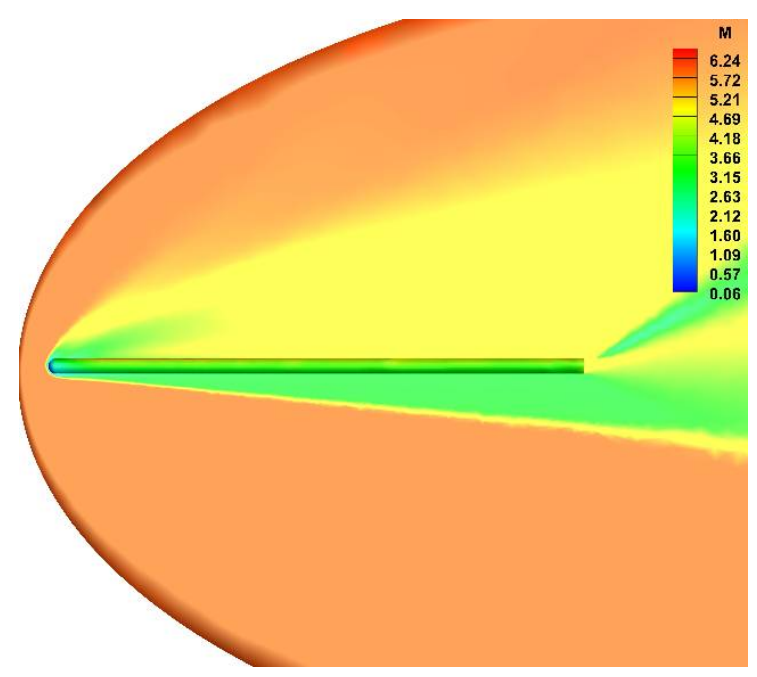

в)

Рис. 5. Поля чисел Маха в центральном осевом сечении: $a-\mathrm{M}=6, \alpha=0^{\circ}$ $\sigma-\mathrm{M}=6, \alpha=10^{\circ} ; \beta-\mathrm{M}=6, \alpha=25^{\circ}$

Из рисунков видно, что важной особенностью газодинамической конфигурации поля течения является закономерное уменьшение угла наклона головной ударной волны при увеличении скорости полета.

Рисунки 6, 7, $a-8$ дают представление о том, как изменяются поля температур и давлений с изменением угла атаки в центральном осевом сечении. Заметно, что с ростом угла атаки возрастает давление и температура в донной части крыла, но не дает представления об их распределении. 


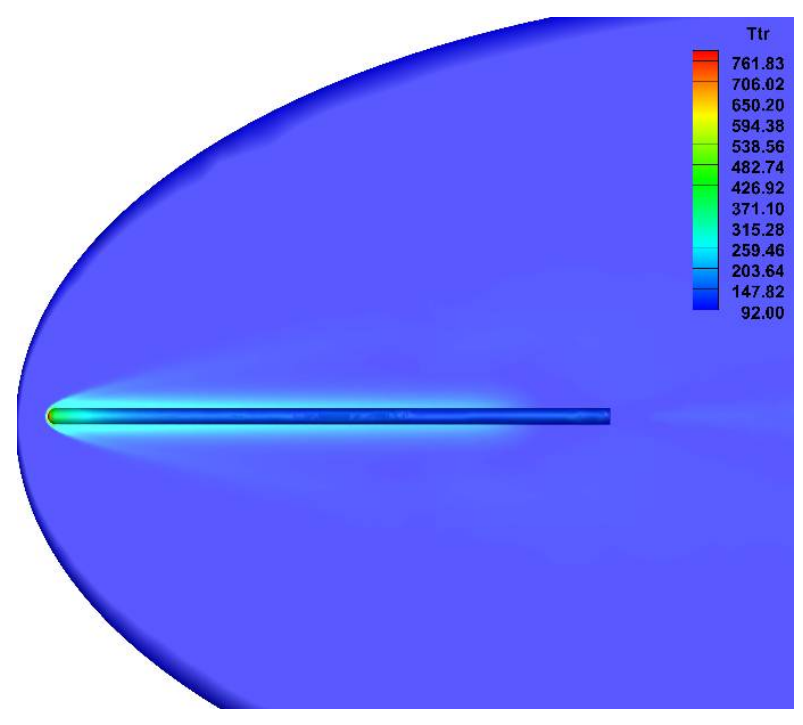

a)

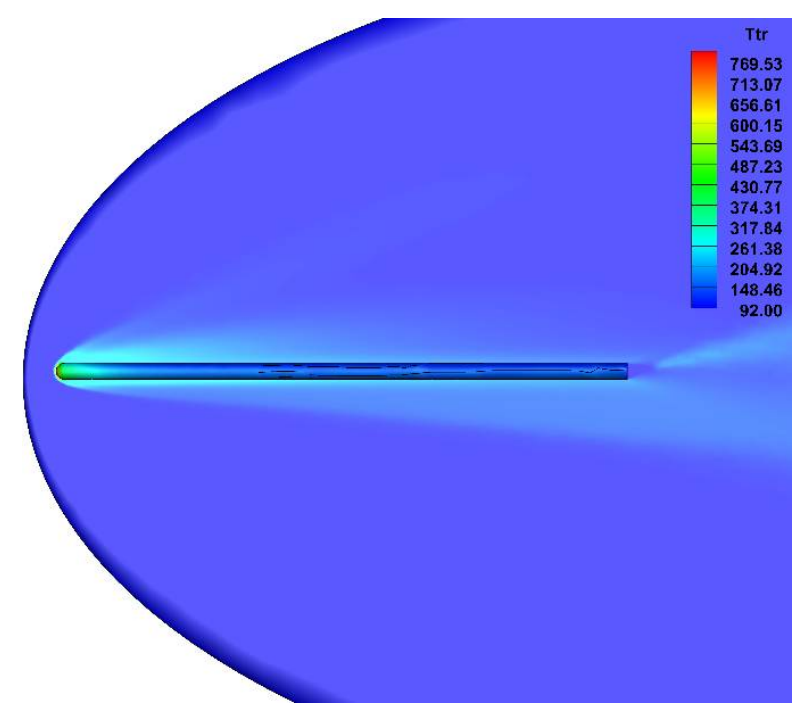

б)

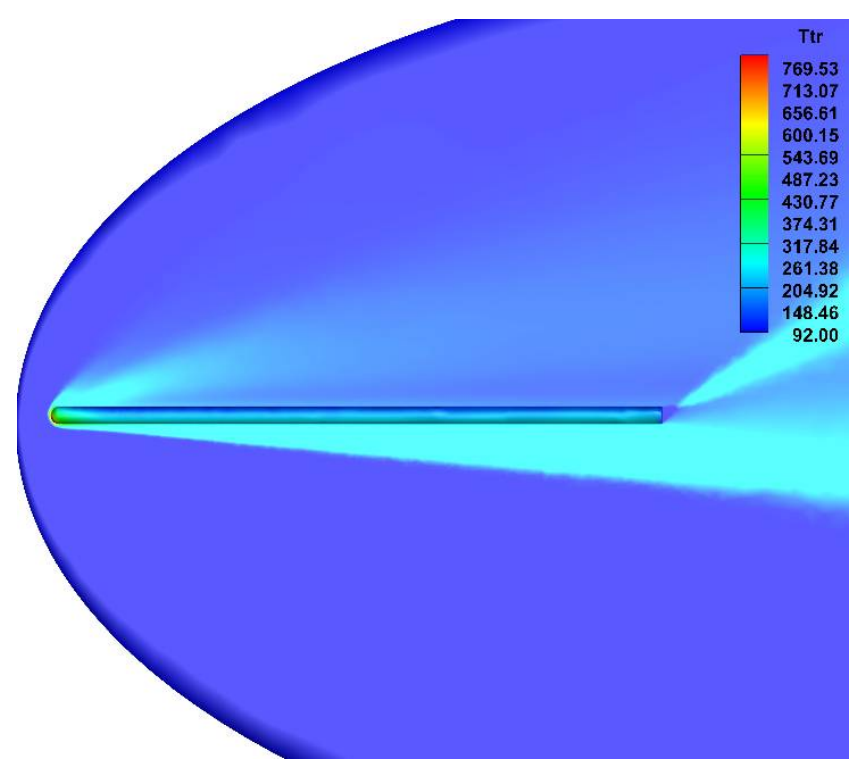

в)

Рис. 6. Поля температур в центральном осевом сечении: $a-\mathrm{M}=6, \alpha=0^{\circ}$ $\sigma-\mathrm{M}=6, \alpha=10^{\circ} ; \beta-\mathrm{M}=6, \alpha=25^{\circ}$ 


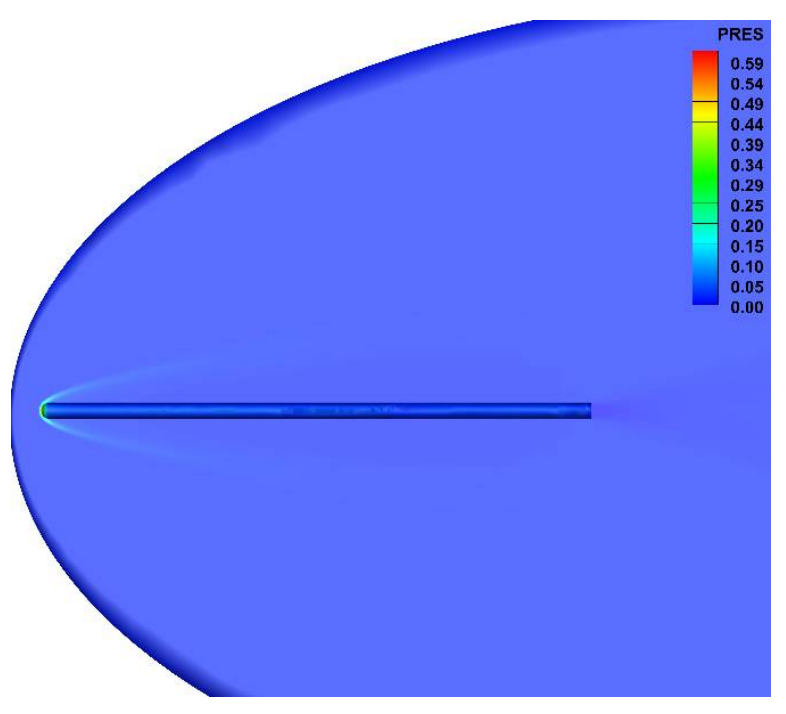

a)

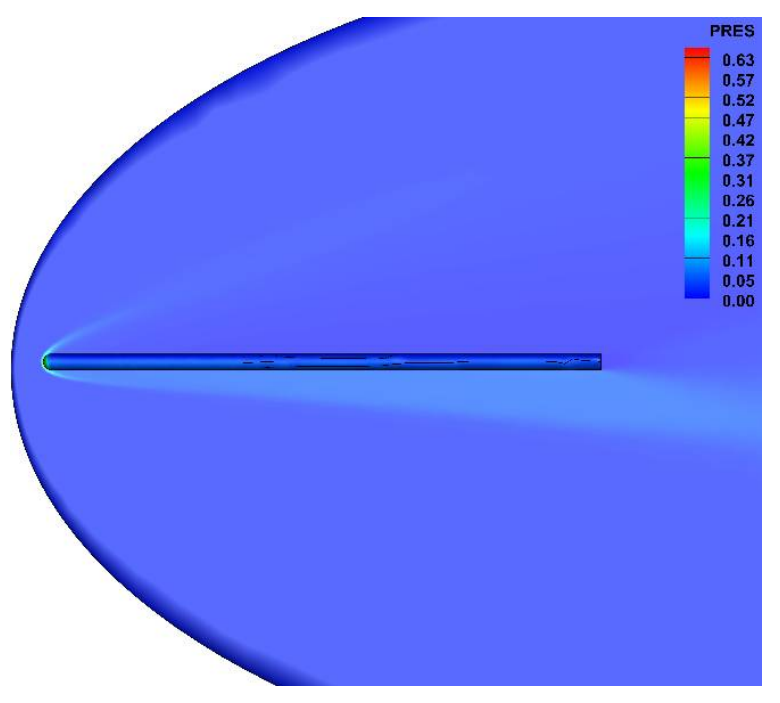

б)

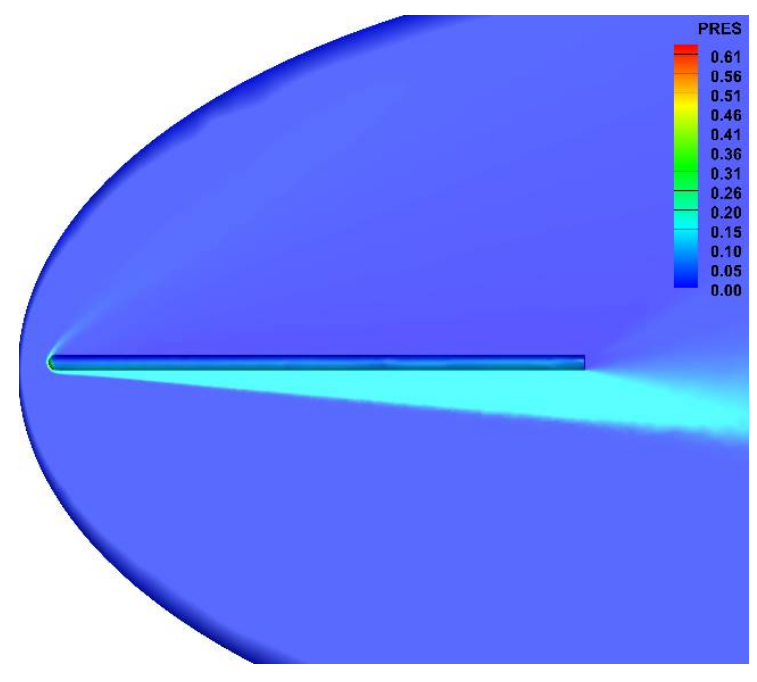

в)

Рис. 7. Поля давления в центральном осевом сечении: $a-\mathrm{M}=6, \alpha=0^{\circ}$ $\sigma-\mathrm{M}=6, \alpha=10^{\circ} ; в-\mathrm{M}=6, \alpha=25^{\circ}$

Рисунок 8, $a-e$ показывает, как изменяется теневая картина при обтекании сложной пространственной модели ГЛА при вариации угла атаки и Маха. Как и прежде, из рисунков видно, как изменяется ударная волна при изменении угла атаки, но в целом картина от изменения числа Маха практически не меняется. 


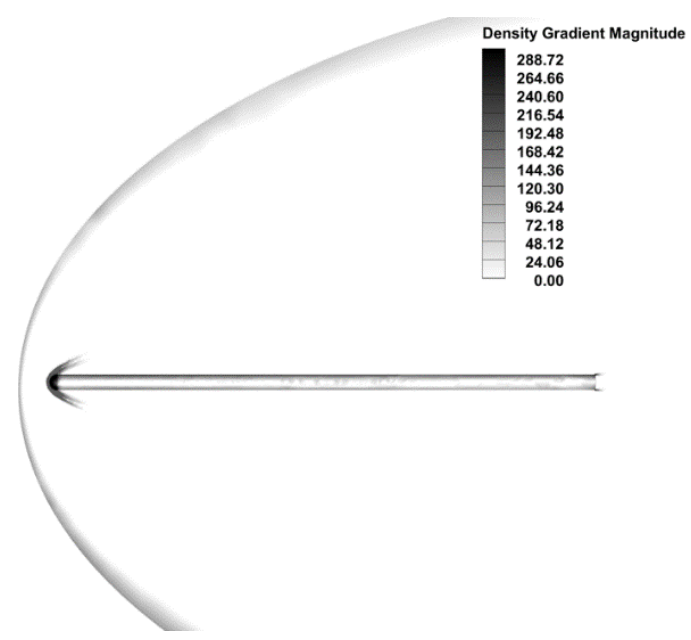

a)

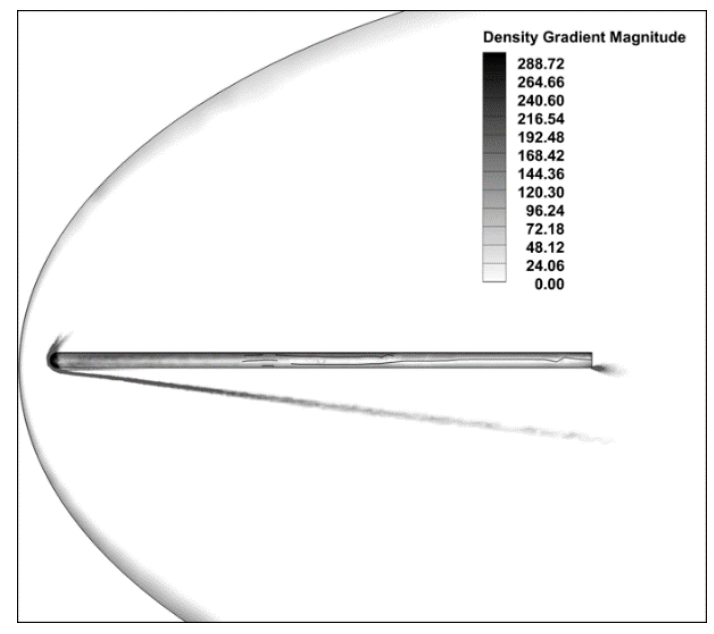

в)

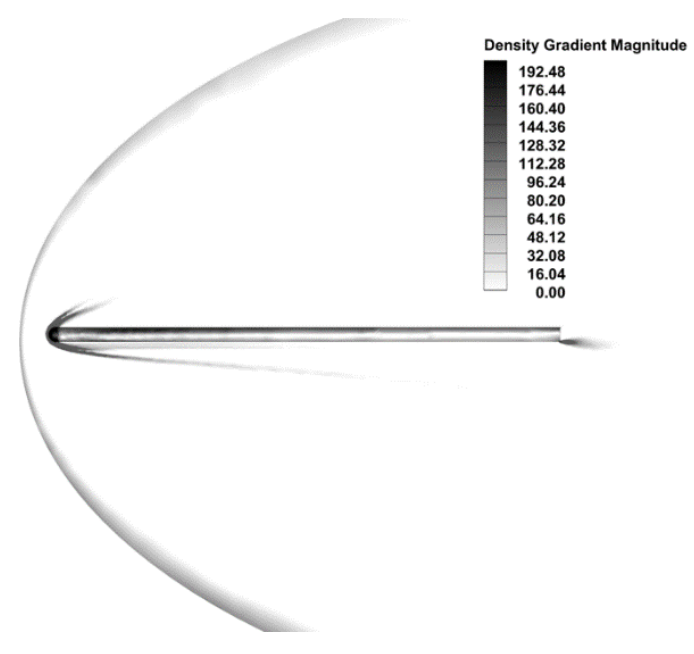

d)

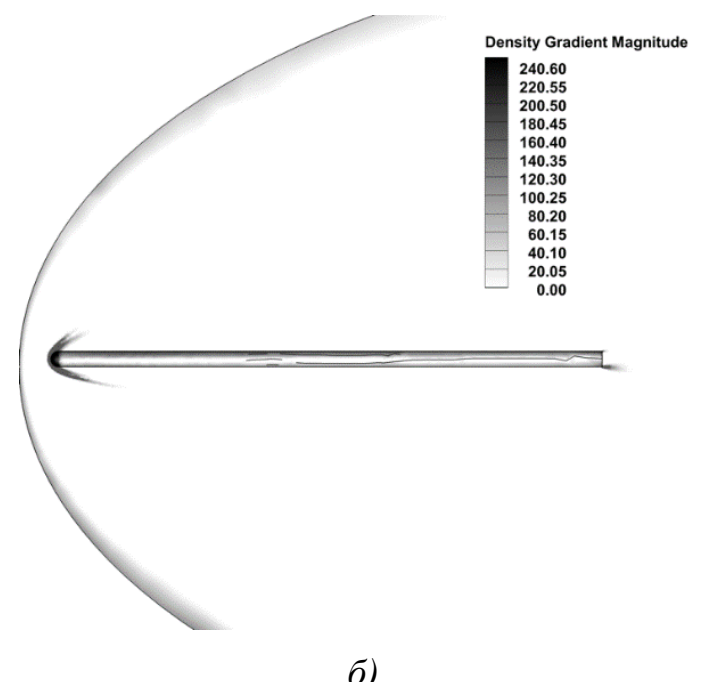

б)

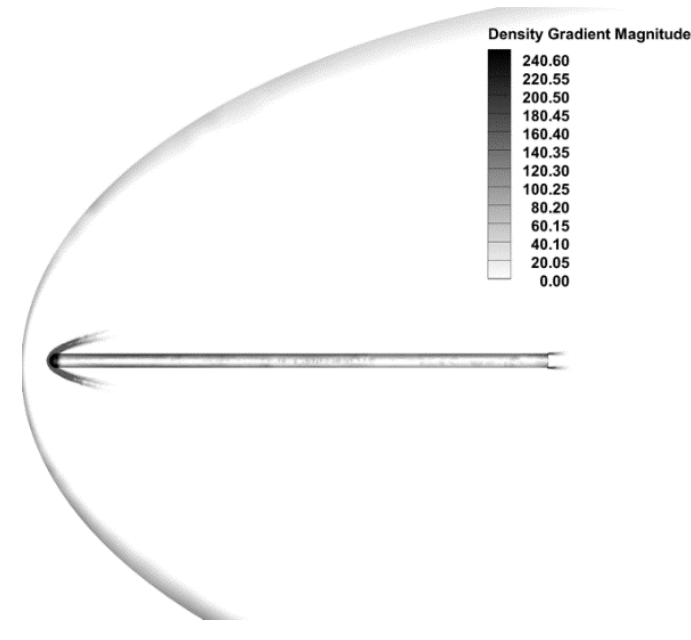

2)

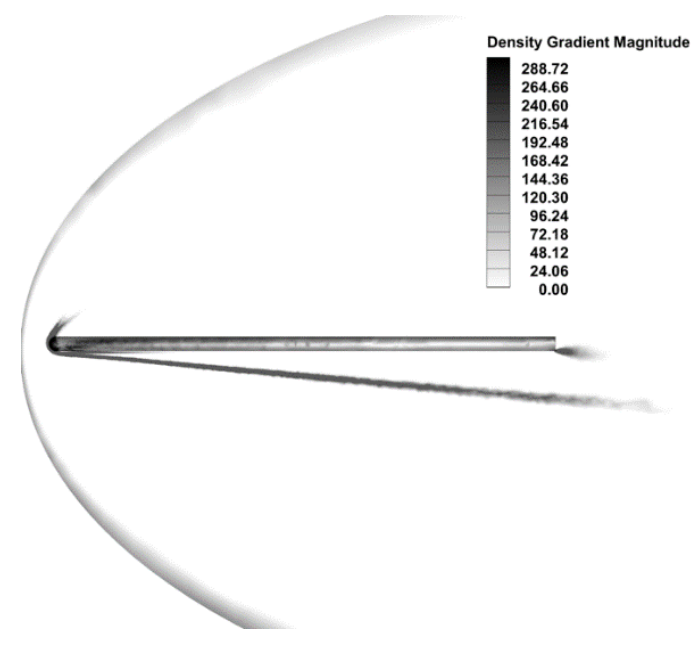

e)

Рис. 8. Теневая картина в центральном осевом сечении: $a-\mathrm{M}=4, \alpha=0^{\circ}$ $\sigma-\mathrm{M}=4, \alpha=10^{\circ} ; в-\mathrm{M}=4, \alpha=25^{\circ} ; 2-\mathrm{M}=6, \alpha=0^{\circ}, \partial-\mathrm{M}=6$, $\alpha=10^{\circ}, e-\mathrm{M}=6, \alpha=25^{\circ}$ 
Кроме вычисления локальных характеристик теплообмена и силового воздействия потока на аппарат, для каждого из рассмотренных режимов обтекания вычислялись интегральные аэродинамические характеристики модели, коэффициенты подъемной силы $C_{y}$, коэффициенты силы лобового сопротивления $C_{x}$. Исследовались зависимости данных характеристик от угла атаки.

На рис. 9 представлены рассчитанные зависимости коэффициентов подъемной силы $C_{y}$, коэффициентов силы лобового сопротивления $C_{x}$ крыла от угла атаки при $\mathrm{M}=6$. В диапазоне от $0^{\circ}$ до $25^{\circ}$ с ростом угла атаки значения коэффициентов возрастают. Дополнительно были проведены расчеты с более подробной сеткой на 3 миллиона ячеек со сгущением вокруг всего крыла. Использованный генератор неструктурированных сеток не позволяет получить вложенность более подробной сетки относительно оригинальной, поэтому сходимость оценивалась по значениям аэродинамических коэффициентов. Как показали исследования (рис. 9), значения коэффициентов не сильно отличаются при использовании более подробной сетки.

На данном этапе в расчетах не учитывалось влияние сил трения на значения аэродинамических коэффициентов. Но для модели столь удлиненной конфигурации их влияние может быть заметным. Поэтому в дальнейшем планируется провести численный анализ влияния сил трения на значения аэродинамических коэффициентов в зависимости от удлинения модели.

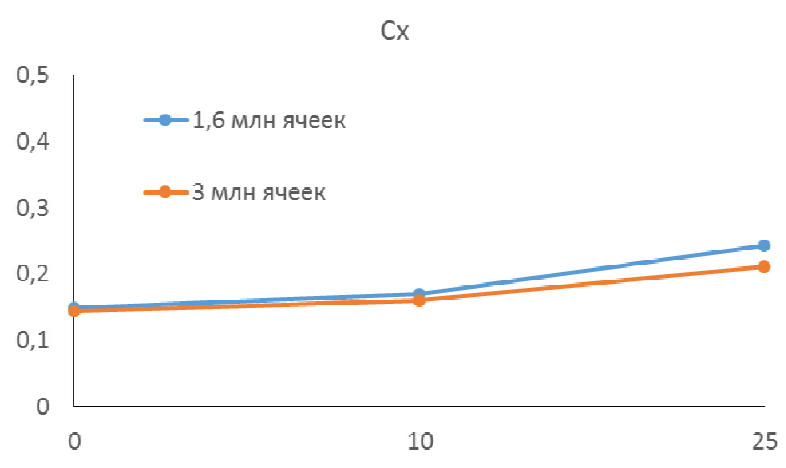

a)

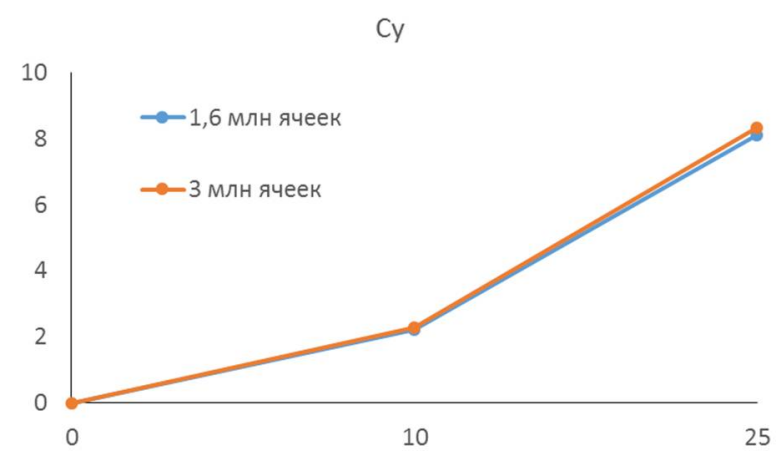

б)

Рис. 9. Зависимость коэффициентов силы лобового сопротивления (a) и подъемной силы (б) от угла атаки

Для условий полета при $\mathrm{M}=6$ и $\alpha=10^{\circ}$ проведено сравнение расчетных и данных в [14]. Результат сопоставления тепловых потоков вдоль поверхности крыла, полученных методами численного моделирования и из [14] показан на рис. 10. Как видно из рисунка удовлетворительных качественного и количественного согласий достигнуто не было, требуется построение более подробных расчетных сеток.

\section{5. Заключение}

В работе на основе компьютерного моделирования гиперзвукового обтекания модели треугольного крыла исследованы основные закономерности поля течения и аэротермодинамики ГЛА во всей области от головной ударной волны до дальнего следа при различных режимах, отличающихся значениями угла атаки и числами Маха. Расчеты выполнены для чисел Maха $\mathrm{M}=4,6$ и углов атаки от $0^{\circ}$ до $25^{\circ}$. Получено представление о структуре ударных волн, образующихся при обтекании пространственной модели гиперзвукового летательного аппарата.

Для каждого режима вычислены интегральные аэродинамические характеристики модели, коэффициенты подъемной силы $C_{y}$ и коэффициенты силы лобового сопротивления $C_{x}$. 
Проведено сравнение данных численного моделирования, проведенного в ЦАГИ, с результатами численного моделирования. Удовлетворительного соответствия данных получено не было, требуется создание более подробных расчетных сеток.

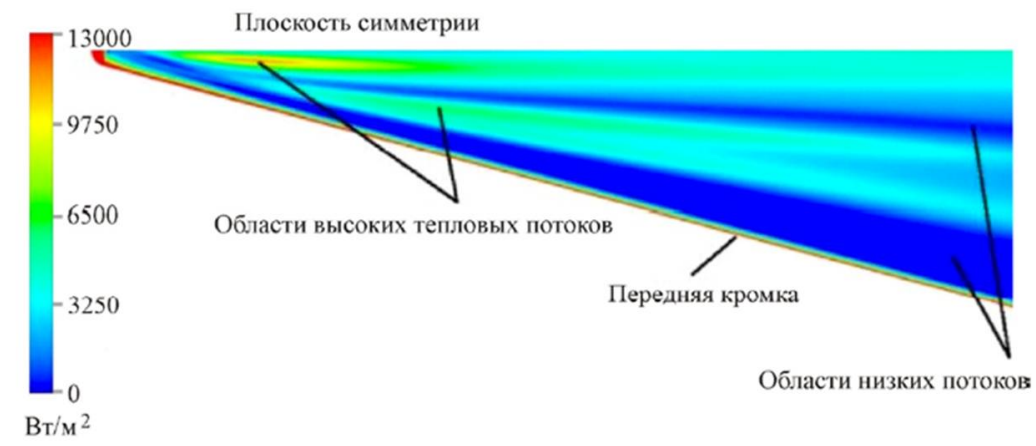

a)

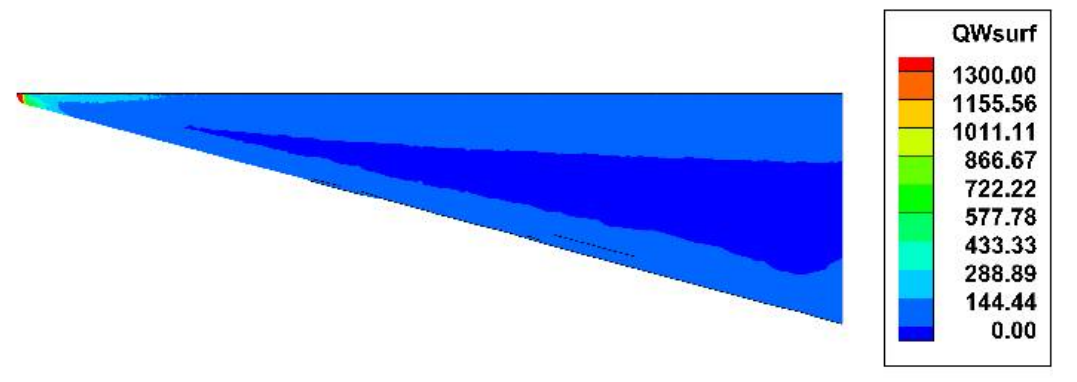

б)

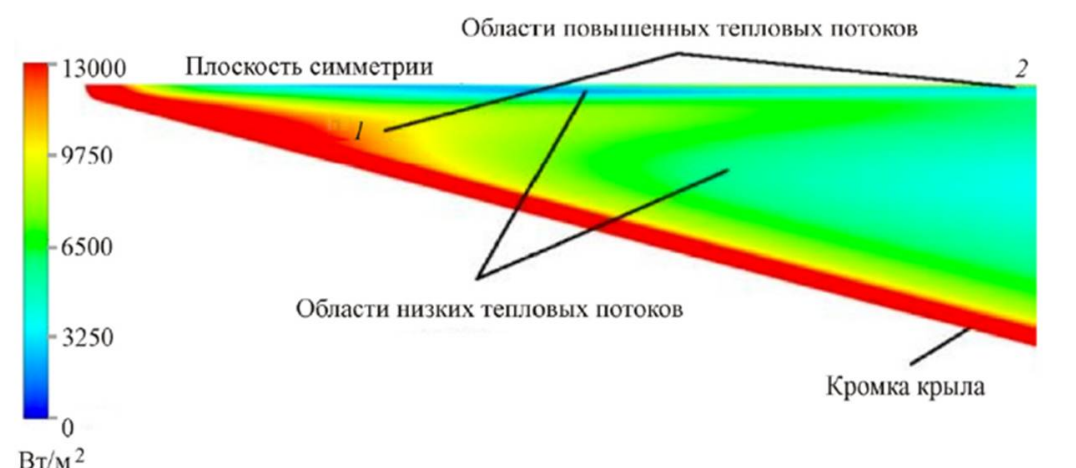

в)

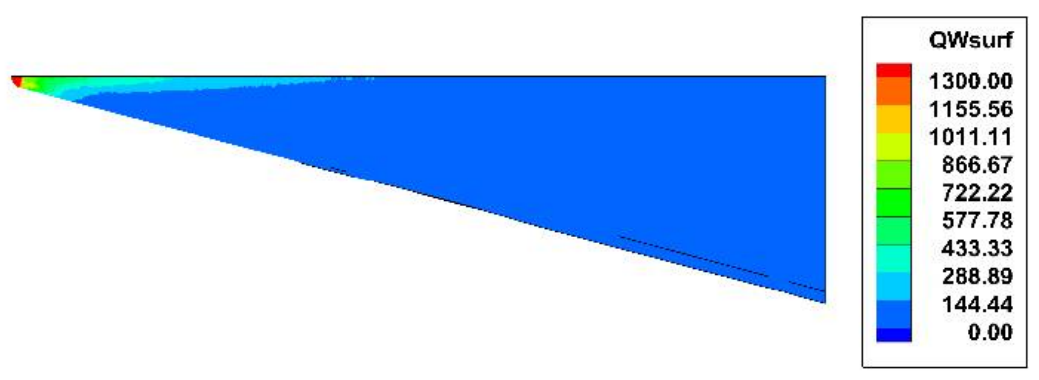

Рис. 10. Распределение теплового потока на крыле при $\alpha=10^{\circ}: a, \sigma$ - подветренная поверхность из [14] и расчетов соответственно; в, 2- наветренная поверхность из [14] и расчетов соответственно

Работа выполнена по теме государственного задания (№ госрегистрации АААА-А17117021310372-6). 


\section{Литература}

1. Нейланд В. Я., Боголепов В. В., Дудин Г. Н., Липатов И. И. Асимптотическая теория сверхзвуковых течений вязкого газа. М.: Физматлит, 2004. 455 с.

2. Лунев В.В. Гиперзвуковая аэродинамика. М.: Машиностроение, 1975. 328c.

3. Anderson J. Hypersonic and High-Temperature Gas Dynamics Second Edition. AIAA Education Series, 2006.

4. Железнякова А.Л., Суржиков С.Т. Применение метода расщепления по физическим процессам для расчета гиперзвукового обтекания пространственной модели летательного аппарата сложной формы//ТВТ. 2013. Т. 51. № 6. С. 897-911.

5. Железнякова А.Л., Суржиков С.Т. Расчет гиперзвукового обтекания тел сложной формы на неструктурированных тетраэдральных сетках с использованием схемы AUSM//TBT. 2014. T. 52. № 2. C. 283-293.

6. Железнякова А.Л., Суржиков С.Т. Численное моделирование гиперзвукового обтекания модели летательного аппарата X-43//Физико-химическая кинетика в газовой динамике. 2011.

T. 11. http://chemphys.edu.ru/issues/2011-11/articles/191/

7. Ermakov M.K., Kryukov I.A. Supercomputer modeling of flow past hypersonic flight vehicles. Journal of Physics: Conference Series. Vol. 815. 012016. 2017.

8. Железнякова А.Л., Суржиков С.Т. Численное моделирование гиперзвукового обтекания цилиндра//Физико-химическая кинетика в газовой динамике. 2008. Т. 7.

http://chemphys.edu.ru/issues/2008-7/articles/470/

9. Суржиков С. Т. Расчетное исследование аэротермодинамики гиперзвукового обтекания затупленных тел на примере анализа экспериментальных данных. М.: ИПМех РАН, 2011. 192 с.

10. Калашников С. В., Кривощапов А. А., Митин А. Л., Николаев Н. В., Расчетные исследования аэродинамических характеристик тематической модели летательного аппарата схемы «летающее крыло» с помощью программного комплекса FlowVision. Компьютерные исследования и моделирование. 2017. Т. 9. № 1. С. 67-74.

11. Давыдов А. А., Луцкий А. Е. Численное моделирование сверхзвукового течения в следе за крылом и его взаимодействия с пересекающимися ударными волнами. М: Препринты ИПМ им. М. В. Келдыша. 2014, № 98, 16 с.

12. Горшков А. Б., Численное исследование вязкого гиперзвукового обтекания треугольного крыла с затупленными кромками. Ж. вычисл. матем. и матем. физ. 2009. Т. 49. № 9. С. 1697-1707.

13. Бабаев Д. А. Обтекание треугольного крыла при больших числах М. Ж. вычисл. матем. и матем. физ. 1963. Т. 3. № 2. С. 397-400.

14. Александров С.В., Ваганов А.В., Шалаев В.И. Физические механизмы образования продольных вихрей, появления зон высоких тепловых потоков и раннего перехода в гиперзвуковом течении около треугольного крыла с затупленными передними кромками. Вестник ПНИПУ. Аэрокосмическая техника. 2016. № 45. С. 9-31.

15. Железнякова А.Л., Суржиков С.Т. Применение метода расщепления по физическим процессам для расчета гиперзвукового обтекания пространственной модели летательного аппарата сложной формы. Теплофизика высоких температур, 2013, том 51, o. 6, с. 897-911.

16. Surzhikov S. T. Validation of computational code UST3D by the example of experimental aerodynamic data. Journal of Physics: Conference Series. Vol. 815. 012023, 2017.

17. Yatsukhno D.S. Numerical simulation of the flow over a hypersonic waverider using the method for splitting into physical processes. Journal of Physics: Conference Series. Vol. 815. 012022, 2017. 